\title{
Investigation of the heat capacity of poly (vinyl methyl ether)/water mixtures using the stepscan method
}

\author{
Lan Guan, Hongyan Xu and Dinghai Huang
}

The heat capacity of poly(vinyl methyl ether) (PVME)/water mixtures was measured over a wide range of temperatures using the stepscan method. The results showed that water exists in different states in the mixtures. The $C_{p}$ values of dry PVME and PVME/ water mixtures with a low water content were obtained by the stepscan method, and the $C_{p}$ of bound water in the mixtures was approximated. The results showed that the $C_{p}$ of bound water in the mixtures with a low water content is higher than that of mixtures with glassy water and ice, and it shows no dependence on water content in the glassy state. Because of the larger conformational contribution to $C_{p}$, the heat capacity of water in the mixtures in the liquid state increases with water content. Because there is a weak interaction between PVME and water molecules, the structure of water in mixtures is different from that of glassy water. The $C_{p}$ value of unfreezable bound water in the mixtures was calculated without considering the effect of polymer content, and the interaction between PVME and water was obtained; these calculations explained the phase separation observed at high temperature.

Polymer Journal (2010) 42, 540-545; doi:10.1038/pj.2010.37; published online 5 May 2010

Keywords: DSC; glassy water; phase separation; stepscan

\section{INTRODUCTION}

Water-soluble polymer solutions have recently aroused a great deal of interest among research studies for use in academic and industrial applications. ${ }^{1,2}$ Aqueous polymers have numerous applications in scientific and industrial areas, such as food and/or pharmaceutical applications. Aqueous polymer solutions have exhibited peculiar and complicated behaviors, such as depressed melting point and crystallization temperature of water in aqueous polymer solutions and unusual phase behavior. At the same time, some aqueous polymer water solutions exhibit phase separations above the lower critical solution temperature and closed loop phase behavior, which are characteristics of polymer solutions that exhibit hydrogen bonding. ${ }^{3,4}$

Poly(vinyl methyl ether) (PVME) is a classical example of a polymer presenting an lower critical solution temperature at a moderate temperature range of around $35^{\circ} \mathrm{C}, 5,6$ above which the solution separates into two phases. It has been shown that, in aqueous solution, the ether functions of repeat units in the PVME molecule form a complex with water molecules. Maeda ${ }^{7}$ proposed a formation of a stable molecular complex with 2.7 molecules of water per repeat unit of PVME. At a higher overall water content, a higher degree of hydration was achieved with up to five water molecules per repeat unit of PVME.

Water-soluble polymer solutions have also been investigated at low temperatures. ${ }^{5,8,9}$ In several systems, it has been found that water did not crystallize when the polymer concentration was high enough. Many theories have been proposed to explain this phenomenon, such as the effect of capillary condensation, the confinement of water clusters by polymer chains, ${ }^{10,11}$ the strong interactions between the highly dipolar water molecules and the polar sites of the hydrophilic polymers $^{12-14}$ and the effect of vitrification of the highly concentrated and viscous polymer solutions. ${ }^{8}$ However, vitrification is not a suitable explanation for aqueous mixtures of PVME because the glass transition temperature of PVME is quite low $\left(\mathrm{ca} .-30^{\circ} \mathrm{C}\right)$ and vitrification is likely to have no effect on the crystallization of water. ${ }^{15,16}$ In noncrystallizable solutions, water is assumed to be bound to polymer chains and a polymer/solvent complex forms. It remains unclear whether this complex is kinetically or thermodynamically controlled. ${ }^{15}$ Further work indicated that, for $w_{\mathrm{PVME}}=0.7$, water crystallized in solutions when ice nuclei were added. ${ }^{17}$ This experiment demonstrated that the complex is thermodynamically unstable and is only observed under some kinetic conditions, and the inhibited crystallization of water in high-polymer-concentration mixtures is related to crystal nucleation instead of to the existence of a stable intermolecular complex.

Although many studies have been conducted on the heat capacity of phase separation in PVME/water mixtures, relatively less work has been reported on the heat capacity of PVME/water mixtures with a low water content in a low temperature range and in the demixing temperature range. This situation is different in that the water in mixtures with a low water content is unable to crystallize, and only a glass transition was detected at low temperature. The water in these mixtures is similar to glassy water. Therefore, this paper investigates 
the heat capacity of water in PVME/water mixtures to understand the thermodynamic behavior of water in polymer/water mixtures.

\section{EXPERIMENTAL PROCEDURE}

\section{Materials}

PVME and water for high-performance liquid chromatography (residue $<0.0003 \%$ ) were purchased from Sigma-Aldrich (St Louis, MO, USA), and used without further purification. The number-average and weight-average molecular weight were determined by gel permeation chromatography as $M_{\mathrm{w}}=20 \mathrm{~kg} \mathrm{~mol}^{-1}$ and $M_{\mathrm{n}}=10 \mathrm{~kg} \mathrm{~mol}^{-1}$, respectively, for a polydispersity index of $M_{\mathrm{w}} / M_{\mathrm{n}}=2$. To obtain dry PVME, the solution was heated at $60^{\circ} \mathrm{C}$ for about $1 \mathrm{~h}$, and then placed in a vacuum oven at $60^{\circ} \mathrm{C}$ for $8 \mathrm{~h}$, ensuring that no water remained.

\section{DSC measurements}

Differential scanning calorimeter (DSC) samples (3-6 mg) of PVME/water mixtures with low water concentrations were prepared by adding determined amounts of PVME and water directly to aluminum sample pans. Excess water was allowed to evaporate slowly at room temperature to obtain samples with a predetermined water content by weight. Subsequently, the samples were quickly hermetically sealed in the aluminum pans and stored at room temperature for several days before analysis to allow them to equilibrate and produce homogeneous mixtures. The sample weight was checked before and after calorimetric analysis to ensure the integrity of the hermetic seal.

Calorimetric measurements were carried out with a PerkinElmer Diamond DSC (PerkinElmer, Waltham, MA, USA) equipped with liquid nitrogen. Highpurity indium and $\mathrm{Hg}$ were used for temperature calibration and an indium standard was used for calibration of heat flow. The stepscan measurement procedures were similar to those of previously reported methods. ${ }^{18,19}$ The stepscan method consisted of multiple heating/isothermal segments through the temperature range of interest. Because both heat capacity equilibration and DSC equilibration are rapid, $C_{p}$ calculation is assumed to be independent of kinetic processes. The temperature step was approximately at $1{ }^{\circ} \mathrm{C}$, with isothermal periods of $2 \mathrm{~min}$ at each temperature; the heating rate was $5{ }^{\circ} \mathrm{Cmin}^{-1}$. The temperature at which $C_{p}$ attained a value halfway between the extrapolated liquid and glassy state values was $T_{\mathrm{g}}$.

\section{RESULTS AND DISCUSSION}

\section{Variation in $C_{p}$ of PVME/water mixtures with temperature}

Figure 1 shows the $C_{p}$ of a PVME/water mixture with water content $=60 \%$ at different temperatures. These traces exhibit two endothermic peaks that are attributed to free water and freezable bound water, respectively. At the low temperature of about $-33^{\circ} \mathrm{C}$, there is a glassy transition, which is the $T_{\mathrm{g}}$ of the PVME-rich amorphous phase in the mixtures. There is evident phase separation at around $35^{\circ} \mathrm{C}$. The stepscan method yielded an accurate result for the phase separation temperature because it allowed enough time for water to crystallize during the heating process. The stepscan method used consisted of multiple heating/isothermal segments through the temperature range of interest. Because both heat capacity equilibration and DSC equilibration are rapid, $C_{p}$ calculation is assumed to be independent of kinetic processes. The $C_{p}$-temperature relationship of a PVME/water mixture with water content $=60 \mathrm{wt} \%$ shows that there are different states of water in the mixture and, at high temperature, there is an evident phase separation. At low temperature, PVME and unfrozen water form an amorphous phase, corresponding to the glass transition. ${ }^{17}$

When the water concentration was low, the water was unable to crystallize and showed melting behavior in aqueous polymer solutions, and only a glass transition could be detected by calorimetric analysis. To investigate the mechanism that presents the crystallization of water, it is necessary to understand the thermodynamics of water and the interaction between PVME and water in soluble polymer

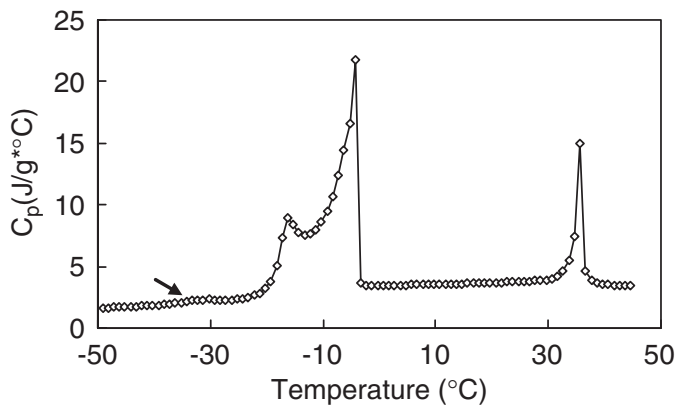

Figure 1 The $C_{p}$ of a PVME/water mixture with a water content of 60 wt $\%$. Glass transition is indicated.

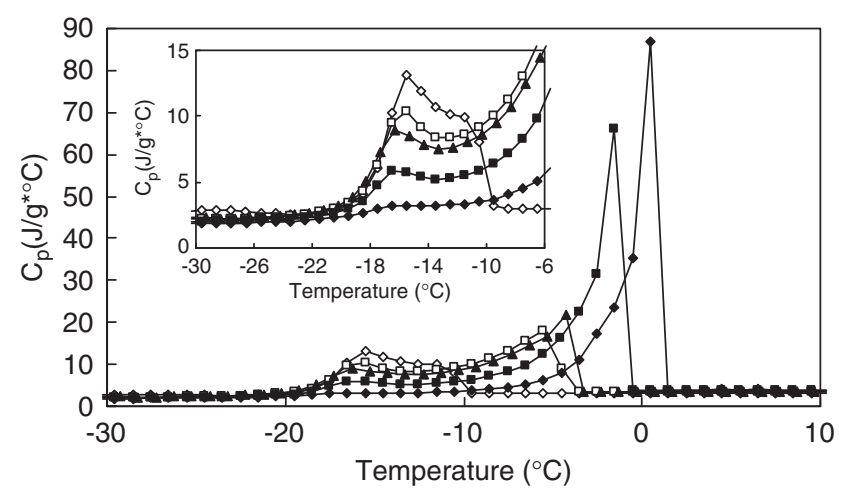

Figure 2 The heat capacity $\left(C_{p}\right)$ of PVME/water mixtures. Water contents are $\diamond, 40 \% ; \square, 55 \% ; \boldsymbol{\Delta}, 60 \% ; \boldsymbol{\square}, 80 \% ; \diamond, 90 \%$.

solutions. The stepscan method was used to investigate the heat capacity of PVME/water mixtures over the whole range of composition. Figure 2 displays the dependence of $C_{p}$ on temperature; the peak melting point $T_{\mathrm{m}}$ of the larger peak increases with water concentration, whereas the $T_{\mathrm{m}}$ of the smaller peak does not increase with water concentration. The two melting phenomena are detected for all samples with a water concentration greater than $40 \mathrm{wt} \%$, which indicates that there are different states of water in those solutions. This is quite different from Loozen's theory, ${ }^{20}$ which proposed that the double melting endoderm in DSC is not associated with the different melting temperatures of various states of water but simply reflects the peculiar shape of the melting line. However, the stepscan method includes melting and recrystallization traces, which allows the mixtures to reach complete crystallization. Therefore, the two melting peaks observed here in PVME/water mixtures are considered to be signals of different states of water in solutions. In addition, the dependence of the $T_{\mathrm{m}}$ of the two melting peaks on water concentration shows that the crystals formed by free water are affected by the polymer content, which reveals that some part of the crystals of free water may come from the water attached to the hydrophobic sites of polymer chains, and the polymer content in the mixtures could change the size of the ice crystals. The water loosely bound to the polar site of polymer chains may form an unstable crystallized structure. These crystals have a constant size because of the interaction between the polymer and water molecules; hence, the ice crystal size could not increase with total water concentration.

Figure 3 shows the experimental $C_{p}$ of PVME/water solutions and the theoretical $C_{p}$ value at $20^{\circ} \mathrm{C}$. The heat capacity of a liquid is the sum of vibrational and conformational contributions. The heat 


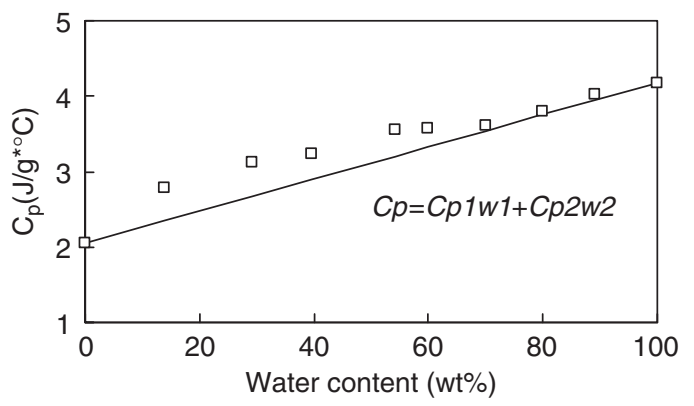

Figure 3 The heat capacity of PVME/water mixtures at $20^{\circ} \mathrm{C}$.

capacity of the mixed system of amorphous polymer and small molecules is based on the following equation: ${ }^{21,22}$

$C_{p}($ poly-sm $)=C_{\text {vib }}($ poly-sm $)+C_{\text {conf }}($ poly-sm $)+C_{\text {ext }}($ poly-sm $)$

where $C_{p}$ (poly-sm) represents the total heat capacity of the system at constant pressure, $C_{\text {vib }}$ (poly-sm) is the vibrational heat capacity at constant volume, $C_{\text {ext }}$ (poly-sm) represents the external heat capacity and $C_{\text {conf }}($ poly-sm) is the conformational heat capacity.

The major part of heat capacity is a result of vibrational contribution. Theoretical heat capacity is approximated by the following equation:

$$
C_{\text {vib }}(\text { poly-sm })=C_{p}^{\text {poly }}(\text { vib }) X_{\text {poly }}+C_{p}^{\mathrm{sm}}(\mathrm{vib}) X_{\mathrm{sm}}
$$

where $X_{\text {poly }}$ and $X_{\mathrm{sm}}$ are the molar fraction of polymer repeating units and small molecules, respectively. Here, the specific heat capacity normalized by weight percent and the specific heat capacity normalized by molar fraction were calculated by multiplying heat capacity by the weight unit and molar fraction of water. These normalized values could be compared with similar data in literature; hence, the weight fractions of polymers and small molecules were treated as $X_{\text {poly }}$ and $X_{\mathrm{sm}}$. Therefore, to a first approximation, the total heat capacity of the PVME-water system is simply predicted as follows:

$$
\begin{gathered}
C_{p}^{\text {PVME-water }}(T)^{\text {solid }}=X_{\mathrm{PVME}} C_{p}^{\mathrm{PVME}}(T)^{\text {solid }}+X_{\text {water }} C_{p}^{\text {water }}(T)^{\text {glassy }} \\
C_{p}^{\text {PVME-water }}(T)^{\text {liquid }}=X_{\mathrm{PVME}} C_{p}^{\mathrm{PVME}}(T)^{\text {liquid }}+X_{\text {water }} C_{p}^{\text {water }}(T)^{\text {liquid }}
\end{gathered}
$$

Equation (3) was used to calculate the heat capacity of amorphous and liquid water in the PVME/water mixtures with different compositions. The experimental results deviate from the theoretical prediction when the water composition is lower than $70 \mathrm{wt} \%$, and as the water concentration decreases, the deviation becomes larger. Amorphous PVME and water form a complex that has a higher $C_{p}$ when the PVME composition is relatively high. Because there are hydrogen bonds and associations in these complexes, the $C_{p}$ of the PVME-rich phase is larger than the theoretical value. In the high-water-composition mixtures, the polymer-rich phase content is small and most of the water molecules in the solutions are free water; hence, the $C_{p}$ of the mixtures is nearly the same as the theoretical predictions.

\section{Heat capacity of PVME/water mixtures during phase separation}

Figure 4 shows $C_{p}$ normalized by PVME weight and it indicates a different dependence on water content. The slope of the dependence decreases in the intermediate concentration range that corresponds to the $C_{p}$ maximum. When the water content is higher than $40 \%$, freezable water appears in the mixtures. With a further increase in water content, free water exists in the mixtures based on $C_{p}$ temperature dependence. Because free water has weak or no interactions with

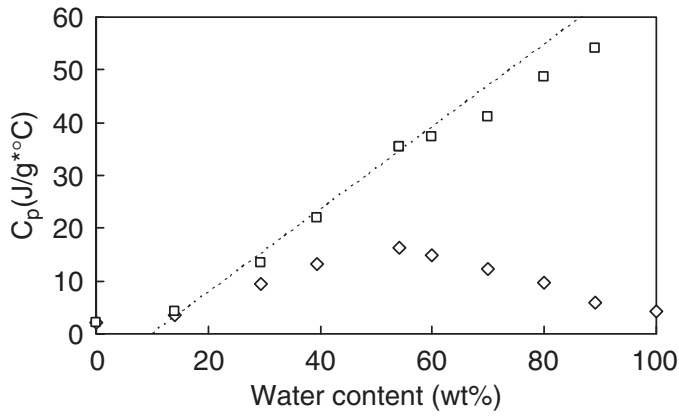

Figure 4 The peak values of the heat capacities of PVME/water mixtures at phase separation. $\diamond$, Per gram of PVME/water solution; $\square$, per gram of PVME.

PVME, this part of the water does not contribute to the demixing enthalpy. The demixing enthalpy decreases, hence the relationship between the $C_{p}$ of mixtures normalized by PVME weight and water content shows a turning point, and $C_{p}$ increases slowly with water content. This indicates that the available water molecules exceed the number necessary for the hydration of the polymer chains with a high water content. ${ }^{23}{ }^{1} \mathrm{H} \mathrm{NMR}^{24}$ reveals that the phase separation in dilute PVME solutions is related to rapid dehydration of polymer chains, and for semidilute and concentrated solutions, it shows that the bound water is more slowly released from PVME globular structures that become more compact with time, and most of the bound water is inside phase-separated structures. ${ }^{25}$ Thus, the bound water is largely responsible for the phase separation.

\section{Heat capacity of PVME/water mixtures with a low water content} at low temperature

The heat capacity of PVME was also detected. Figure 5 shows the $C_{p}$ temperature dependence. The experimental data are in agreement with the data in the literature. ${ }^{26}$ The glass transition temperature of PVME was determined in this study to be about $-28.6{ }^{\circ} \mathrm{C}$. Figure 6 presents the data for heat capacities of polymer solutions at low temperature. Glass transition significantly decreases with increasing water content. When the water content is higher than $40 \%$, melting peak appears in the DSC results, showing that freezable water exists in the mixtures. Water is a universal and effective plasticizer for the soluble polymer, with a reported $T_{\mathrm{g}}$ of $135.9 \mathrm{~K},{ }^{27}$ which lowers the glass transition temperature of polymer materials. Water molecules bound to polymer chains were unable to move freely and crystallize into ice crystals during cooling. The heat capacity results show that the water can decrease the glass transition of PVME, functioning as a plasticizer as a result of the strong hydrogen bonds between PVME chains and water molecules. The glass transition temperature of the PVME/water mixtures can be estimated from the change in the specific heat capacity at the transition temperature using the halfheight method; the experimental $T_{\mathrm{g}}$ results are shown in Figure 7. Using the stepscan method avoided the common phenomenon of an enthalpy overshoot observed at $T_{\mathrm{g}}$.

The fitting results of the Fox equation and the Gordon-Taylor equation are also given in Figure 7. The Fox equation ${ }^{28}$ is expressed as

$$
1 / T_{\mathrm{g}}=w_{1} / T_{\mathrm{g} 1}+w_{2} / T_{\mathrm{g} 2}
$$

and the Gordon-Taylor equation ${ }^{29}$ is as follows:

$$
T_{\mathrm{g}}=\left(w_{1} T_{\mathrm{g} 1}+k T_{\mathrm{g} 2}\right) /\left(w_{1}+k w_{2}\right)
$$

where $w_{1}, w_{2}$ and $T_{\mathrm{g} 1}, T_{\mathrm{g} 2}$ are the weight fractions and $T_{\mathrm{g}}$ values of water and polymer, respectively, and $k$ is an adjustable parameter. 


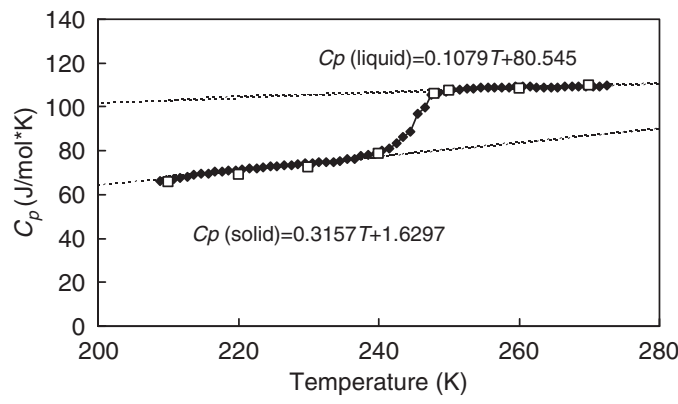

Figure 5 The experimental heat capacities of solid and liquid PVME. ४, Experimental data; $\square$, experimental data from the reference. ${ }^{26}$ The upper and lower solid lines indicate the fitted linear functions of the solid and liquid PVME heat capacities, respectively.

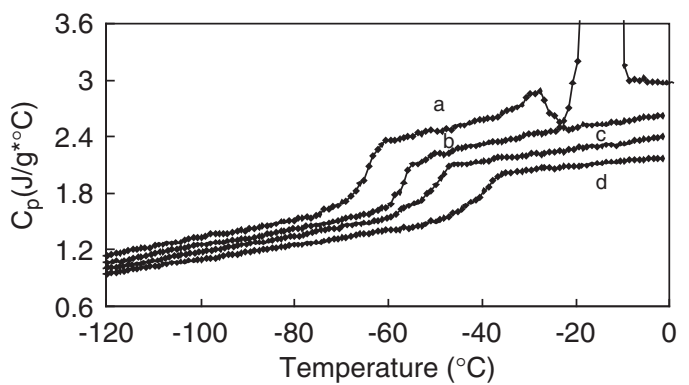

Figure 6 The heat capacity of PVME/water measured at low temperature. The water contents are (a) 40wt\%; (b) 30wt\%; (c) 20wt\%; (d) $10 w t \%$.

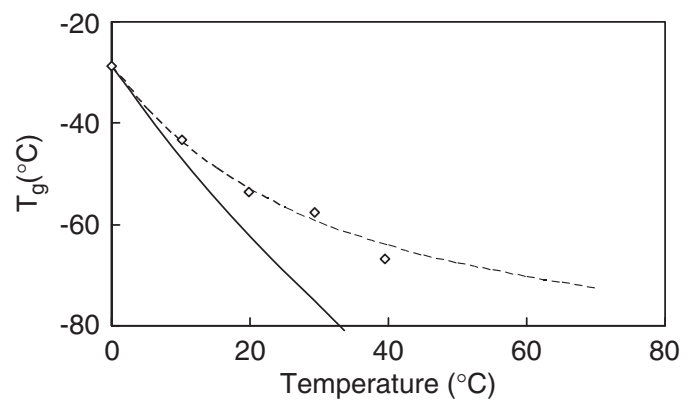

Figure 7 The glass transition temperature $\left(T_{\mathrm{g}}\right)$ of PVME/water mixtures with a low water content. $\diamond$, Experimental result; solid line, Fox equation; dashed line, Gordon-Taylor equation ( $k=0.245)$.

Miscible blends show $k$-values not far from unity, whereas too high or too low values result in limited miscibility. The fitting parameter $k$ is 0.245 in our experiment, indicating that the PVME/water mixture with a low water concentration is a thermodynamically miscible blend. The Gordon-Taylor equation gives a much better fitting result than the Fox equation.

The fitting functions of solid and liquid state PVME were calculated over the investigated temperature range to obtain the heat capacity of water in polymer solutions at low temperature. The $C_{p}$ values of solid and liquid water in the PVME/water solutions were approximated by equation (3) and the results are presented in Figures 8 and 9. The $C_{p}$ calculation of solid water here is significantly higher than the experimental $C_{p}$ of crystal ice and amorphous ice in the references, ${ }^{30,31}$ indicating that in solid-state PVME/water mixtures, water interacts with polymer through hydrogen bonds and could not form ice crystals. The water is in a solid state similar to glassy water. However,

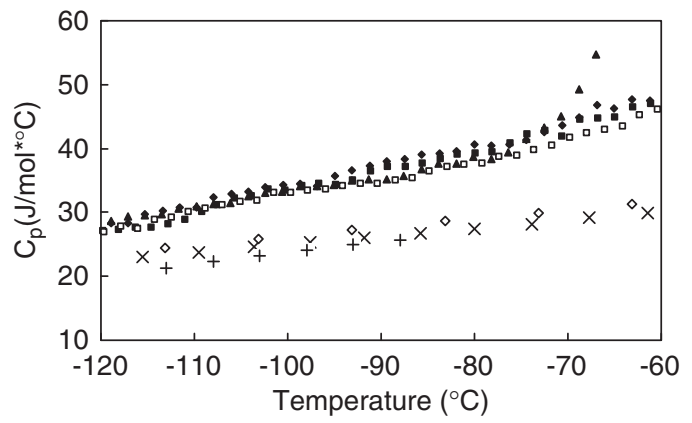

Figure $\mathbf{8}$ The calculated heat capacity of bound water of PVME/water mixtures in the glassy state at low temperature. The water contents are $\downarrow$, $10 \% ; \boldsymbol{\square}, 20 \% ; \square, 30 \% ; \boldsymbol{\Delta}, 40 \% ; \times$, experimental $C_{p}$ of ice in reference ${ }^{30}+$, experimental $C_{p}$ of amorphous ice in reference; ${ }^{31} \diamond$, heat capacity of glassy water in reference. ${ }^{21,22}$

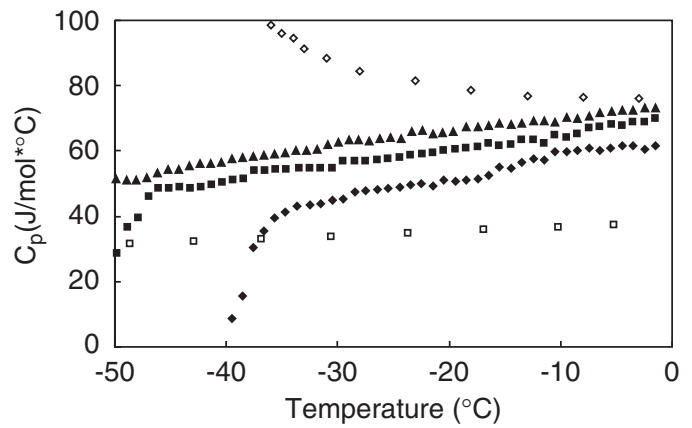

Figure 9 Calculated heat capacity of bound water of PVME/water mixtures in liquid state. Water contents are $\bullet, 10 \% ; \boldsymbol{\square}, 20 \% ; \boldsymbol{\Delta}, 30 \%$; $\square$, experimental $C_{p}$ of ice in reference; ${ }^{30} \diamond$ : experimental $C_{p}$ of supercooled water in reference. ${ }^{32}$

the $C_{p}$ of water in the mixtures is higher than that in glassy water. $^{21,22,30}$ The calculated $C_{p}$ values of water in polymer solutions with different water concentrations are nearly the same, which reveals that the state of the water in low-water-concentration solutions does not depend on the water concentration. The $C_{p}$ calculation of liquid water is shown in Figure 8 and reports that the calculated $C_{p}$ of liquid water is lower than the $C_{p}$ of supercooled water ${ }^{32}$ and this value increases with water concentration. The calorimetric behavior of liquid-state water in polymer solutions is different from that of bulk water below $0{ }^{\circ} \mathrm{C}$, owing to the hydrogen bonds between PVME chains and water molecules, which break the hydrogen bonding between water molecules and affect the structure of water molecules. The calculation of $C_{p}$ in mixtures with different water concentrations may be different because in the liquid state the conformational contribution to heat capacity becomes more evident than in the solid state. A high water content produces more conformational heat capacity, therefore the water in the liquid state increases with water concentration. This result indicates that the interaction between PVME and water molecules and polymer content have an effect on the $C_{p}$ calculation of water, which produces a deviation from the result of equation (3a).

A new method was used to calculate the $C_{p}$ of water in mixtures to eliminate the effect of the interaction between water and polymer. The heat capacities of PVME/water mixtures at $-100{ }^{\circ} \mathrm{C}$ were obtained using the stepscan method, and the $C_{p}$-water concentration relationship is shown in Figure 6. The $C_{p}$ results of low-water-concentration mixtures with a water content $\leqslant 40 \%$ were fitted, the relation 


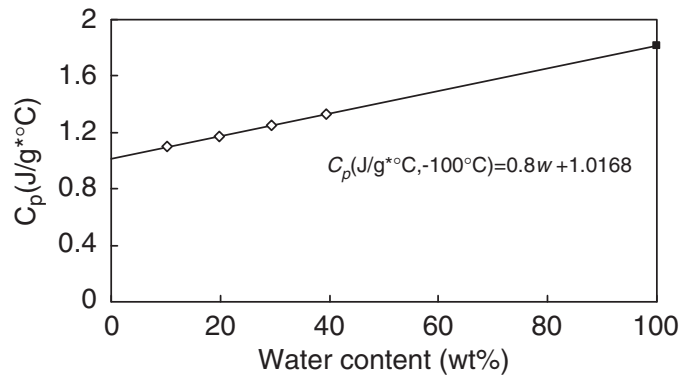

Figure 10 The experimental $C_{p}$ of a PVME/water mixture with a low water content at $-100^{\circ} \mathrm{C}$. $\diamond$, experimental result; $\mathbf{\square}, C_{p}$ value of bound water in the mixtures calculated by extrapolating the fitting result of experimental values to a water content of $100 \%$.

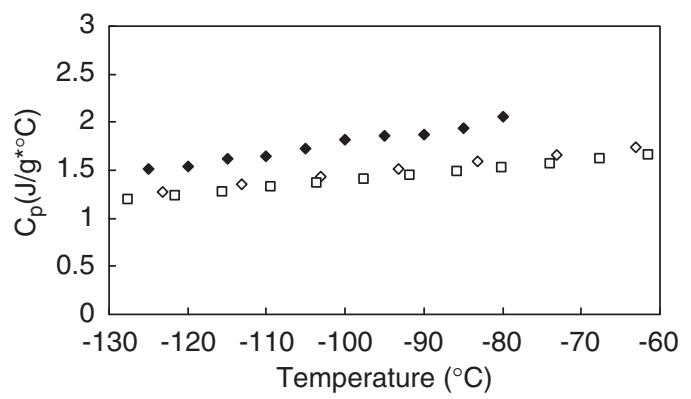

Figure 11 The calculated $C_{p}(\bullet)$ of unfreezable bound water in PVME/water mixtures at low temperature. $\diamond$, experimental $C_{p}$ of glassy water ${ }^{21} \square, C_{p}$ of ice. ${ }^{30}$

$C_{p}\left(J / g^{* \circ} \mathrm{C},-100{ }^{\circ} \mathrm{C}\right)=0.8 w_{1}+1.0168$ is shown in Figure 10. Mixtures with a water content $\leqslant 40 \%$ are in the completely glassy state at $-100{ }^{\circ} \mathrm{C}$, hence the $C_{p}$ of unfreezable bound water was approximated using the linear fitting relation approximately and $C_{p}=1.8168 \mathrm{~J} / g^{\star \circ} \mathrm{C}$ at a water content of $100 \%$. The $C_{p}$ of unfreezable bound water at other low temperatures was obtained by fitting a linear $C_{p}$-water composition dependence and extrapolating it to a water content of $100 \%$ in a similar method. Figure 11 shows the calculated value and the results from related references. The figure shows that the calculated value of the heat capacity of unfreezable bound water in PVME/water mixtures is higher than that of glassy water in other studies in a low temperature range. ${ }^{21,30}$ With increasing temperature, the calculated value increases and the difference from previous results becomes greater. This result shows that, as a result of the weak interaction between PVME and water molecules, water mobility in PVME/water mixtures is higher than that in other hydrophilic polymer/water mixtures. At low temperature, there is only a glassy transition in the mixture with a low water content. The vibrational contribution of water to heat capacity is larger owing to the weak hydrogen bonding interaction. Therefore, the calculated value of $C_{p}$ for bound water in the mixtures is higher than those in literature. This result indirectly indicates the weak interaction between PVME and water molecules and could explain why there is a phase separation of PVME/water mixtures at $35^{\circ} \mathrm{C}$.

\section{CONCLUSION}

The heat capacities of PVME/water mixtures were measured over a wide temperature range by the stepscan method. The $C_{p}$ value of mixtures reaches a maximum in the intermediate water content range, consistent with the dependence of demixing enthalpy on water content. The investigation of the $C_{p}$ of PVME/water mixtures over a wide temperature range shows that different states of water exist in the mixtures. The $C_{p}$ values of dry PVME and PVME/water mixtures with a low water content were obtained by the stepscan method, and the $C_{p}$ of bound water in the mixtures was approximated. Results show that, in the glassy state, the $C_{p}$ of bound water in the mixtures with a low water content is higher than that of glassy water and ice, and $C_{p}$ does not depend on water content. It has been proposed that this unfreezable bound water is more mobile than glassy water and that the interactions between PVME and water molecules are weak. In the liquid state, owing to the larger conformational contribution to $C_{p}$, the heat capacity of water in mixtures increases with increased water content. By extrapolating the experimental $C_{p}$ of mixtures to a water content of $100 \%$, the $C_{p}$ of unfreezable bound water was approximated without considering the effect of hydrogen bonding and polymer content, which is evidently greater than that previously reported. Because there is a weak interaction between PVME and water molecules, the structure of water in the mixtures is different from that of glassy water and it has a larger mobility, hence the calculated $C_{p}$ value is higher than that of glassy water. The heat capacity result shows that water in the mixtures with a low water content is evidently different from that of glassy water, because of the weak interaction between PVME and water. The calculation of the $C_{p}$ value of unfreezable bound water indirectly confirms this weak interaction.

1 Stillinger, F. H. in Water in polymers (ed. Rowland, S. P.) (American Chemical Society, Washington DC, 1980).

2 Schulz, D. N. in Water-soluble polymers (eds. Shalaby, S. W., McCormick, C. L., Butler, G. B.) Vol. 467 (ACS Symposium Series; American Chemical Society, Washington, DC, 1991).

3 Walker, J. S. \& Vause, C. A. Theory of closed-loop phase diagrams in binary fluid mixtures. Phys. Lett. A. 79A, 421-424 (1980).

4 Dormidontova, E. E. The role of competitive PEO-water and water-water hydrogen bonding in aqueous solutions of PEO. Macromolecules 35, 987-1001 (2002).

5 Maeda, Y. IR spectroscopic study on the hydration and the phase transition of poly(vinyl methyl ether) in water. Langmuir. 17, 1737-1742 (2001).

6 Horne, R. A., Almeida, J. P., Day, A. F. \& Yu, N. T. Macromolecule hydration and the effect of solutes on the cloud point of aqueous solutions of polyvinyl methyl ether: a possible model for protein denaturation and temperature control in homeothermic animals. J. Colloid Interf. Sci. 35, 77-84 (1971).

7 Maeda, H. Interaction of water with poly(vinyl methyl ether)in aqueous solution. J. Polym. Sci., Part B: Polym. Phys. 32, 91-97 (1994).

8 Meeussen, F., Bauwens, Y., Moerkerke, R., Nies, E. \& Berghmans, H. Molecular complex formation in the system poly(vinyl methyl ether)/water. Polymer 41, 3737-3743 (2000).

9 De Dood, M. J. A., Kalkman, J., Strohhofer, C., Michielsen, J. \& Van der Elsken, J. Twocomponent system cycloheptanol (C7) plus cyclooctanol (C8): an extraordinary system. J. Phys. Chem. B 107, 5906-5913 (2003).

10 Cuperus, F. P., Bargeman, D. \& Smolders, C. A. Critical points in the analysis of membrane pore structures by thermoporometry. J. Membr. Sci. 66, 45-53 (1992).

11 Modesti, M., Dall'Acqua, C., Lorenzetti, A. \& Florian, E. Mathematical model and experimental validation of water cluster influence upon vapour permeation through hydrophilic dense membrane. J. Membr. Sci. 229, 211-223 (2004).

12 Filho, G. R. \& Bueno, W. A. Water states of Cuprophan (hemodialysis membrane). J. Membr. Sci. 74, 19-27 (1992).

13 Scherer, J. R., Bailey, G. F., Kint, S. R. Y., Malladi, D. P. \& Bolton, B. Water in polymer membranes. 4. Raman scattering from cellulose acetate films. J. Phys. Chem. 89, 312-319 (1985).

14 Ping, Z. H., Nguyen, Q. T., Chen, S. M., Zhou, J. Q. \& Ding, Y. D. States of water in different hydrophilic polymers-DSC and FTIR studies. Polymer 42, 8461 (2001).

15 Maeda, Y., Mochiduki, H., Yamamoto, H., Nishimura, Y. \& Ikeda, I. Effects of ions on two-step phase separation of poly(vinyl methyl ether) in water as studied by IR and Raman spectroscopy. Langmuir. 19, 10357-10360 (2003).

16 Moerkerke, R., Koningsveld, R., Berghmans, H., Dusek, K. \& Solc, K. Phase transitions in swollen networks. Macromolecules 28, 1103-1107 (1995).

17 Zhang, J. M., Berge, B., Meeussen, F., Nies, E., Berghmans, H. \& Shen, D. Y. Influence of the interactions in aqueous mixtures of poly(vinyl methyl ether)on the crystallization behavior of water. Macromolecules 36, 9145-9153 (2003).

18 Huang, D., Simon, S. L. \& McKenna, G. B. Chain length dependence of the thermodynamic properties of linear and cyclic alkanes and polymers. J. Chem. Phys. 122 084907-1-84907-6 (2005). 
19 Huang, D., Simon, S. L. \& McKenna, G. B. Equilibrium heat capacity of the glass- forming poly(a-methyl styrene) far below the Kauzmann temperature: the case of the missing glass transition. J. Chem. Phys. 119, 3590-3593 (2003).

20 Loozen, E., Durme, K. V., Nies, E., Mele, B. V. \& Berghmans, H. The anomalous melting behavior of water in aqueous PVME solutions. Polymer 47, 7034-7042 (2006).

21 Pyda, M. Conformational contribution to the heat capacity of the starch and water system. J. Polym. Sci. Part B Polym. Phys. 39, 3038-3054 (2001).

22 Pyda, M. Conformational heat capacity of interaction systems of polymer and water. Macromolecules 35, 4009-4016 (2002)

23 Durme, K. V., Assche, G. V., Nies, E. \& Mele, B. V. Phase transformations in aqueous low molar mass poly(vinyl methyl ether) solutions: theoretical prediction and experimental validation of the peculiar solvent melting line, bimodal LCST and (adjacent) UCST miscibility gaps. J. Phys. Chem. B. 111, 1288-1295 (2007).

24 Spevacek, J., Hanykova, L. \& Starovotova, L. ${ }^{1} \mathrm{H}$ NMR relaxation study of thermotropic phase transition in poly(vinyl methyl ether) $/ \mathrm{D}_{2} \mathrm{O}$ solutions. Macromolecules $\mathbf{3 7}$, 7710-7718 (2004).
25 Spevacek, J. \& Hanykova, L. ${ }^{1} \mathrm{H}$ NMR study on the hydration during temperatureinduced phase separation in concentrated poly(vinyl methyl ether) $/ \mathrm{D}_{2} \mathrm{O}$ solutions. Macromolecules 38, 9187-9191 (2005).

26 Pyda, M., Durme, K. V., Wunderlich, B. \& Mele, B. V. Heat capacity of poly (vinylmethylether). J. Polym. Sci. Part B: Polym. Phys. 43, 2141-2153 (2005).

27 Hansen, E. W., Gran, H. C. \& Sellevold, E. J. Heat of fusion and surface tension of solids confined in porous materials derived from a combined use of NMR and calorimetry. J. Phys. Chem. B. 101, 7027-7032 (1997).

28 Fox, T. G. Influence of diluent and of copolymer composition on the glass temperature of a polymer system. Bull. Am. Phs. Soc. 1, 123 (1952).

29 Gordon, M. \& Taylor, J. S. Ideal copolymers and the second-order transitions of synthetic rubbers I. Non-crystalline copolymers. J. Appl. Chem. 2, 493-500 (1952).

30 Giauque, W. F. \& Stout, J. W. The entropy of water and the third law of thermo-dynamics. The heat capacity of ice from 15 to $273^{\circ} \mathrm{K}$. J. Am. Chem. Soc. 58, 1144-1150 (1936).

31 Handa, Y. P. \& Klug, D. D. Heat capacity and glass transition behavior of amorphous ice. J. Phys. Chem. 92, 3323-3325 (1988).

32 Angell, C. A., Ogunl, M. \& Sichlna, W. J. Heat capacity of water at extremes of supercooling and superheating. J. Phys. Chem. 86, 996-1002 (1982). 